\title{
ENT trainee papers presented at the Summer Meeting of ENT Scotland (Scottish Otolaryngological Society), 8-9 May 2014, Dunblane, Scotland, UK
}

Standardisation of ultrasound scan reporting of thyroid nodules: would an ' $R$ ' status be useful?

A McPhee, V Visvanathan, L Clark

From the Southern General Hospital, Glasgow

\section{Introduction}

Ultrasound imaging is an established tool in the evaluation of thyroid nodules. However, unlike the 'Thy' status, there is no standardisation in ultrasound reporting for these patients.

\section{Aims}

The aims of our project were to study and analyse ultrasound reports of thyroid nodules and delegate a radiology $(\mathrm{R})$ status based on a previously validated tool (thyroid imaging reporting and data system).

Methods

A five-year (from 2007 to 2012) retrospective computer database review was conducted.

Results

The study comprised 173 patients (53 males and 120 females), aged from 21 to 28 years (mean age, 56.43 years). The sensitivity, specificity, and positive and negative predictive values of $\mathrm{R}$ status in detecting malignancy were 57.58 per cent, 92.47 per cent, 73.08 per cent and 86 per cent respectively.

\section{Conclusion}

An R status will be a useful adjunct for patient counselling and treatment planning. It has a high specificity and a 50 per cent chance of positively identifying malignant nodules. We recommend standardisation of ultrasound scan reports and auditing of results.

Complications of ventilation tube insertion: nested case-control comparison of cleft and non-cleft children

I Smillie, S Robertson, A Yule, D M Wynne, C J H Russell From the Yorkhill Hospital, Glasgow

\section{Background}

Optimising hearing in cleft palate patients by early recognition and management of otitis media with effusion is essential for speech development. Past evidence has suggested higher complications from ventilation tube insertion in cleft palate patients, and has led to a trend not to treat these patients surgically. However, previous studies failed to match comparison groups for age and gender.

\begin{abstract}
Methods
The study utilised a nested case-control design, in which cleft and non-cleft children were matched for age and gender. Sixty cleft palate patients underwent ventilation tube insertion between May 2002 and October 2012 at the Royal Hospital for Sick Children, Glasgow. The patients in the non-cleft control group, who were matched in terms of age and gender, were selected from a database of 2943 ventilation tube insertion cases. The outcomes assessed from this data were otorrhoea, attendance at clinic for complications and other complications (not otorrhoea).
\end{abstract}

Results

There was no statistically significant difference in otorrhoea rates between the two groups $(p=0.522)$. However, the noncleft cohort had 151 documented cases of otorrhoea, compared to 121 in the study group (ratio of 1.25:1). Other reported complications were also higher in non-cleft patients, with totals of 43 and 25 respectively (ratio of 1.7:1). There was no difference in ENT clinic attendance for complications $(p=0.659)$

\section{Discussion}

This study indicates that complications are not higher within this treatment group, and therefore cleft patients should be treated with ventilation tube insertion under the same guidance as non-cleft patients. Indeed, there could be an argument for a shift in practice with more aggressive treatment in this patient group that is already vulnerable to speech and social developmental delay.

Are we managing paediatric periorbital cellulitis effectively?

L Hughes, I Khan, H Kubba

From the Yorkhill Hospital, Glasgow

Introduction

Periorbital cellulitis is a common ENT emergency presentation in the paediatric population. Most affected patients are admitted for intravenous antibiotic treatment because of the serious complications associated with this pathology.

\section{Aim}

The study aimed to evaluate our current management of paediatric periorbital cellulitis in Greater Glasgow and Clyde based on established local guidelines.

\section{Methods}

The study involved retrospective data collection (via the Clinical Portal) of patients admitted with periorbital cellulitis 
from March 2012 to March 2013. All paediatric patients coded as 'cellulitis of the face' were initially included in this study. Out of 170 patients coded with this diagnosis, 122 patients were diagnosed with periorbital cellulitis. Discharge letters for these patients were analysed and the relevant data were collected. This included: patient demographics; hospital site; site and grade of infection; in-patient management including antibiotic therapy, decongestant therapy and use of imaging; surgical intervention; ophthalmology review; and discharge medication and follow up.

Results

All 122 patients were admitted. All patients received intravenous antibiotics as recommended by the local guidelines. Only 67 of the 122 patients ( 54.9 per cent) received in-patient nasal decongestants. Only 100 patients (81.9 per cent) received ophthalmology in-patient review. Eight patients required surgical intervention. All patients were discharged on oral antibiotics. Only 64 patients (52.4 per cent) were discharged on nasal decongestants. Eighty-six patients (76.7 per cent) had follow-up appointments. Out of those not followed up, only six patients re-presented, with minor eye complaints.

\section{Conclusion}

Antibiotic stewardship for paediatric periorbital cellulitis is effective in Greater Glasgow and Clyde. Surgical intervention is timely and appropriate. However, improvements need to be made to ensure that ophthalmological review is conducted and nasal decongestants are utilised. Patients managed conservatively do not require routine ENT follow up. This will reduce out-patient waiting times. Strict implementation of local guidelines is required.

Early glottic carcinoma: 10-year single-centre outcome analysis

J C L Yeo $^{1}, \mathrm{~S} \mathrm{Ali}^{1}$, E Junor ${ }^{2}$, A S Evans ${ }^{1}$

From the ${ }^{1}$ Lauriston Building, Edinburgh, and ${ }^{2}$ Western General Hospital, Edinburgh

\section{Background}

The treatment of choice for early glottic cancer remains controversial. Some centres still favour radiotherapy (RT) over surgery. We assessed patients treated for early glottic cancer in the Edinburgh Cancer Centre to compare the differences in outcome dependent on stage and primary treatment modality.

\section{Methods}

A retrospective review was conducted of patients treated for early glottic cancer at the Edinburgh Cancer Centre between 1999 and 2009. Patients were stratified according to clinical stage, stage $1\left(\mathrm{~T}_{1} \mathrm{~N}_{0}\right)$ or $2\left(\mathrm{~T}_{2} \mathrm{~N}_{0}\right)$, and primary treatment modality treatment, either transoral laser resection or RT. The local recurrence rate, salvage treatment rate and fiveyear disease-specific survival rate were assessed.

Results

Between 1999 and 2009, 263 patients were diagnosed with early glottic cancer; 194 patients were stage 1 and 69 were stage 2 . Of those with stage 1 disease, 55 had transoral laser resection and 139 had RT. In the transoral laser resection group, there were seven recurrences (13 per cent); all affected patients underwent subsequent salvage RT. In the RT group, there were 20 recurrences (14 per cent); 15 affected patients underwent salvage laryngectomy. The five-year disease-specific survival rates for the stage 1 transoral laser resection and RT groups were 100 per cent and 96 per cent respectively. In the stage 2 group, 6 had transoral laser resection and 63 had RT. In the stage 2 transoral laser resection group, there were four recurrences. In the RT group, there were 16 recurrences ( 25 per cent); 14 of these patients underwent salvage laryngectomy. The five-year disease-specific survival rates for the stage 2 transoral laser resection and RT groups were 80 per cent and 83 per cent respectively.

\section{Conclusion}

Transoral laser resection and RT give equivalent, excellent rates of cure for stage 1 laryngeal cancer. Disease-specific survival rates for patients with stage 2 laryngeal disease were lower, but comparable to those of other centres. Small numbers of patients treated by transoral laser resection for stage 2 disease preclude meaningful comparison regarding the optimum treatment modality. Transoral laser resection appears to provide comparative local control rates and better laryngectomy-free rates when compared to RT.

Botulinum toxin A for children with salivary control problems: positive predictors for an effective response

J Hendry, J Montgomery, S McCusker, E Lumley, H Kubba From the Royal Hospital for Sick Children, Glasgow

\section{Objective}

This study aimed to review the response of children who received botulinum toxin $\mathrm{A}$ injections for saliva control in our institution.

\section{Methods}

This is a retrospective case series of children who attended a dedicated saliva control clinic in a paediatric tertiary referral hospital. Children were identified from the saliva control database, created from the records of children attending the service since its formation in 2006 . All the children had previously undergone a three-month trial of pharmacological therapy, with no responses. All injections were performed freehand to the parotid and submandibular glands. The children's background diagnosis and co-morbidities, age at presentation, dose of botulinum toxin A, treatment response, and any complications, were recorded.

\section{Results}

Ninety-seven children (59 males; 61 . per cent) were identified. The patients were aged between 2 months and 18 years (mean of 8.6 years; median of 8.8 years). A total of 175 botulinum toxin A doses were administered (mean of 1.9 injections per child). The dose injected varied between 0.52 units $/ \mathrm{kg}$ and 21.28 units $/ \mathrm{kg}$ (mean of 5 units $/ \mathrm{kg}$; median of 4.2 units $/ \mathrm{kg}$ ). This was performed using local anaesthetic in 131 cases ( 75 per cent). The remainder were performed under general anaesthetic. The number of injections received by each child ranged between 1 and 11 (mean rate of 1.9). Responses to botulinum toxin A were classed as effective in 109 cases (62 per cent), partially effective in 14 cases ( 8 per cent) and not effective in 50 cases $(29$ per cent). A further two children were awaiting clinical follow up. The response duration was between 0.25 and 18 months (mean of 4 months). Complications were seen following 22 injections (10.9 per cent). Data modelled using binary logistic regression analysis indicated that male sex and cerebral palsy were predictors of a better response to botulinum toxin $\mathrm{A}$. 


\section{Conclusion}

Botulinum A injected under local anaesthetic is a safe, effective treatment for children with sialorrhoea. The main adverse effect this can have is dysphagia, which is of concern in children that can swallow independently prior to injection. Male sex and cerebral palsy are independent positive predictors of a successful outcome of botulinum toxin A injections for sialorrhoea, which enables targeted patient selection.

Endoscopic drainage of subperiosteal orbital abscess in children: the Aberdeen experience

G Lawson, I Khan, P Asimakopoulos, B Ram

From the Royal Aberdeen Children's Hospital

\section{Introduction}

The exact incidence of orbital complications due to sinusitis in children is unknown. However, a medial subperiosteal orbital abscess is the most common serious complication to occur. Surgical intervention is mandatory whenever antibiotic treatment fails. Most authors prefer open surgical procedures such as external drainage or external ethmoidectomy, while others recommend transnasal endoscopic drainage as the first attempt at sinus decompression. We present our experience and technique for endoscopic drainage.

\section{Objective}

This study aimed to review the safety and efficacy of endoscopic drainage of subperiosteal orbital abscesses secondary to sinusitis, and to evaluate risk factors that may be associated with failure.

\section{Study design}

A retrospective chart review was conducted of case notes and computed tomography (CT) scans of patients who underwent endoscopic drainage of an orbital abscess from 2007 to 2013 at the Royal Aberdeen Children's Hospital.

\section{Results}

The study comprised 13 patients with a mean age of 6 years. All patients had a subperiosteal abscess as confirmed on CT scan (type 3, according to the Chandler classification). Twelve of the 13 subperiosteal orbital abscesses identified, each located medially, resolved without complication after endoscopic drainage. Only one patient needed revision surgery, which involved both internal and external drainage. No major complications were reported. Streptococcus pyogenes was the commonest organism isolated. The average length of hospital stay was 3.2 days.

\section{Conclusion}

Endoscopic drainage of medial subperiosteal orbital abscesses appears to be safe and effective. Based on our experience, the endoscopic transnasal drainage approach is an efficient and safe approach to treat subperiosteal abscesses. The lack of a periorbital scar and reduced length of hospital stay are added advantages when compared to the external approach.

\section{Use of elasticated hooks in rhinology and otology}

P Asimakopoulos, I Khan, B Ram

From the Aberdeen Royal Infirmary

\section{Introduction}

Adequate exposure is essential in both rhinological and otological procedures, which often require appropriate assistance. Quite frequently surgeons lack assistance in the operating theatre and have to depend on the scrub team to overcome these problems.

\section{Methods}

We describe our experience in using elasticated hooks (Lone Star retractor system) in open rhinoplasty and in the postauricular approach for middle-ear surgery.

Results

The Lone Star retractor system was adequately used in: elevation of the columellar flap, lateral crus dissection, mid dorsum exposure and the anterior septal dissection stages during open rhinoplasty. It was also useful in skin retraction during the postauricular approach, and during temporalis fascia and conchal cartilage graft harvesting.

\section{Discussion}

We have found the Lone Star retractor system of elasticated hooks to be a flexible tool. It has the ability to rapidly adapt to changes in the surgical field with minimal effort during the operative procedure, offering optimal visibility and access. There were no complications, such as skin flap tear, cartilage tear or excessive bleeding from the hook insertion sites, in our series.

Post-operative nasal debridement following functional endoscopic sinus surgery: systematic review of the literature

\section{R Green, A Banigo, I Hathorn}

From the St John's Hospital, Livingston

\section{Background}

Post-operative management following functional endoscopic sinus surgery (FESS) varies greatly.

\section{Objective}

The study aimed to establish whether frequent debridement of the nasal cavity improves outcome following FESS.

\section{Evaluation}

Two authors independently reviewed the identified studies using consolidated standards of reporting trials ('CONSORT') guidelines.

Results

Six randomised, controlled trials (RCTs) were identified. Four studies compared debridement against no debridement and two compared the frequency of debridement. Results for 337 patients were included. Visual analogue scales (VAS) and endoscopic scores were employed in all studies. Longterm results showed no difference in Sino-Nasal Outcome Test or endoscopic scores. One study demonstrated improvements in VAS scores at 56 weeks, while others showed shortterm improvement with frequent debridement. Two studies showed that debridement groups suffered more pain.

\section{Conclusion}

There is limited evidence supporting nasal debridement following FESS in improving symptoms. Given the discomfort and cost of performing frequent nasal debridement, there needs to be further well-designed RCTs showing clear benefits to support this approach. 
Clinical outcomes of adenoid cystic carcinoma of the head and neck in a single centre over a 21-year period

T Magos, J C L Yeo, S Ali, E Junor

From the Edinburgh Cancer Centre

\section{Background}

Adenoid cystic carcinoma is a rare salivary gland malignancy with a good five-year survival rate. It has a long natural history, and frequently re-presents with late local recurrences and distant metastasis.

\section{Method}

All cases of head and neck adenoid cystic carcinoma treated at the Edinburgh Cancer Centre between 1992 and 2013 were identified and analysed.

\section{Results}

The data for 51 patients were analysed. The mean length of follow up was 102 months. Forty-three per cent of cases involved minor salivary glands and 31 per cent involved the parotid gland. Histology was solid in 15 per cent of cases, tubular in 26 per cent and cribriform in 59 per cent. Tumour (T) stage was a significant predictor for survival. The 10-year overall survival rates were 92 per cent for stage I and II disease and 50 per cent for stage III and IV disease. Mean time to recurrence was 56 months.

\section{Conclusion}

Tumour stage is a predictor of survival. Late recurrences are not uncommon. Adenoid cystic carcinoma remains a difficult disease to treat. It has a high tendency to recur even if radical excision and adjuvant therapy has been performed.

\section{Reduction thyroplasty in the transgendered patient}

\section{J Montgomery, K MacKenzie}

From the Glasgow Royal Infirmary

Transgendered patients represent a small but significant number of patients attending otolaryngology services. In our clinic, we are referred patients enrolled in the male to female gender reassignment programme for a small part of their feminisation surgery: to reduce the external appearance of the laryngeal cartilages. Reduction thyroplasty, often called chondrolaryngoplasty or referred to colloquially as 'tracheal shave', is one of the earliest surgical procedures requested by transgender patients. We discuss the otolaryngology-related issues of transgendered patients and provide details of the surgical technique.

In the past 5 years, 14 patients have undergone reduction thyroplasty in our department. A detailed pre-operative discussion is essential to ensure expectations are adequately addressed. All patients are admitted to a single room. No vocal fold modifications are made during the procedure. We have had no serious complications, but one patient had some wound tethering post-operatively which required referral to plastic surgery.

\section{Round window niche dimensions: cadaveric bones study}

M Shakeel, P Spielmann, S Jones, S S M Hussain

From the Ninewells Hospital and University of Dundee Medical School

\section{Objectives}

The study aimed to measure the various dimensions of the round window niche, focusing on the bony overhang and the vertical height between the free margin of the bony overhang and floor of the niche.

\section{Methods}

Fourteen formalin-preserved human temporal bones were dissected to expose the round window niche. The niche area was filled with a soft mould material, which was removed once it had solidified, demonstrating the shape of the round window niche. The procedure was repeated to ensure reliability. Three measurements were taken: (1) the depth of the niche extending from the free margin of the bony overhang to the fundus; (2) the vertical height from the free margin of the overhang to the floor of the niche (entrance); and (3) the anteroposterior dimension of the niche.

\section{Results}

Based on the mould technique, the three dimensions were calculated as follow: bony overhang $=2.1 \mathrm{~mm}$ (range, $1.9-2.2 \mathrm{~mm}$ ), entrance to niche $=2 \mathrm{~mm}$ (range, $1.8-2.1 \mathrm{~mm}$ ) and anteroposterior length $=3.9 \mathrm{~mm}$ (range, $3.3-4.95 \mathrm{~mm})$.

\section{Conclusion}

Our data confirm that the bony overhang must be removed in Vibrant Soundbridge implantation, while carrying out round window vibroplasty, in order to achieve good placement of the floating mass transducer and round window coupler against the round window membrane.

Surgical management of spontaneous cerebrospinal fluid leaks involving the temporal bone

M I Syed, B Kuske, D Summers, P F Statham, D W Sim

From the Western General Hospital, Edinburgh

\section{Objectives}

The study aimed to describe the patient demographics and modalities of presentation, and review the techniques in the surgical management of spontaneous cerebrospinal fluid (CSF) otorrhoea.

\section{Design}

A retrospective review was conducted at a tertiary referral centre.

\section{Materials and methods}

Patients with spontaneous CSF otorrhoea, who were managed surgically during the last 20 years, were included in the study. Clinical case notes and operative notes were reviewed to obtain information on patient demographics, clinical presentation, investigations, operative techniques and post-operative follow up.

\section{Results}

Fifteen patients, who underwent 16 procedures, were included in the review. The mean age of presentation was 52 years; the male to female ratio was $4: 11$. The clinical presentations were varied, and included otorrhoea, recurrent meningitis, hearing loss, otorhinorrhoea and pneumocephalus. A combined mastoidectomy and middle fossa craniotomy was performed using a combination of allogenic and autologous materials in all cases.

\section{Conclusion}

A joint (otolaryngology and neurosurgery) combined approach provides adequate and safe exposure, with several 
advantages; in our series, there was a 100 per cent primary success rate.

Percutaneous endoscopic gastrostomy insertion by head and neck surgeons: systematic review

M Bannister, K Ah-See

From the Aberdeen Royal Infirmary

\section{Introduction}

Percutaneous endoscopic gastrostomy ('PEG') is performed to maintain nutrition in patients with head and neck cancer. The procedure is increasingly performed by head and neck surgeons. We discuss a systematic review of the literature to assess the effectiveness of gastrostomy insertion by this group of doctors.

\section{Materials and methods}

A literature search of the Embase, PubMed and Cochrane Collaboration databases was performed on 15th February 2014, using the keywords 'percutaneous gastrostomy', 'PEG', 'placement' and 'insertion' with 'head and neck cancer', 'patient', 'laryngeal carcinoma', 'oral cavity carcinoma', 'oropharyngeal carcinoma', 'thyroid carcinoma' and 'hypopharyngeal carcinoma'. The number of insertions, and the success and complication rates, were recorded.

Results

A total of 381 article abstracts were reviewed. Ten articles were included in the review, involving 979 patients from 1990 to 2008. The percutaneous endoscopic gastrostomy insertion experiences of ENT surgeons (eight articles) and oral and maxillofacial surgeons (two articles) have been reported. The average percutaneous endoscopic gastrostomy insertion failure rate was 3 per cent (range, $0-7$ per cent). The average rate of major complications was 3 per cent (range, $0-8$ per cent); the minor complication rate was 14 per cent, with a greater range $(6-35$ per cent $)$.

\section{Discussion}

Percutaneous endoscopic gastrostomy tube insertion by head and neck surgeons is internationally established. Percutaneous endoscopic gastrostomy insertion is commonly performed in combination with surgery by this group, either at examination under anaesthesia or during tumour resection. Insertion success and complication rates are comparable to those of gastroenterologists and general surgeons.

\section{Conclusion}

Percutaneous endoscopic gastrostomy insertion by ENT surgeons can be safe and as successful as insertion performed by surgeons from other specialties. By providing a percutaneous endoscopic gastrostomy insertion service, ENT surgeons can reduce waiting times for oncology patients requiring insertion.

Are any investigations required in acute tonsillitis?

M Bannister, P Chakravarty

From the Aberdeen Royal Infirmary

\section{Introduction}

Acute tonsillitis is a common emergency presentation in ENT. It is principally a clinical diagnosis based on patients' symptoms and tonsil appearance. The need for investigations can be questioned. We assess the influence of haematology, biochemistry, blood culture, monospot and throat swab investigations on management decisions and patient outcomes.

\section{Materials and methods}

A retrospective review was conducted of case notes and discharge summaries of patients admitted to our hospital ward during a 90-month period between May 2006 and November 2013. Records were reviewed to assess if and how any investigations altered patient management or led to atypical care interventions.

\section{Results}

A total of 152 patients' case notes were reviewed. Investigations appeared to have little influence on patient management. Urea and electrolyte results influenced the degree of intravenous fluid therapy provided in three patients (2 per cent). Monospot testing for Epstein-Barr virus infection only guided aftercare advice provision, not the treatment provided. Positive monospot results correlated with the clinical diagnosis in 71 per cent of admissions with glandular fever. No haematology, liver function, C-reactive protein, blood culture or throat swab results influenced patient management or outcome.

\section{Discussion}

The only regular investigation that may be required for tonsillitis patients is monospot testing $(p=0.07)$. Urea and electrolyte testing did not influence in-patient stay in dehydrated patients $(p=0.008)$.

This study suggests that significant healthcare savings can be attained without affecting patient care. Based on our regional costing index of emergency admission laboratory tests, omitting investigations that did not influence patient management in any admission would have saved $£ 5302$ in this group of patients.

\section{Conclusion}

Based on our study, only a very limited requirement exists for investigations in cases of acute tonsillitis. Clinical diagnosis alone is the most reliable guide for treatment.

Vestibular schwannoma growth patterns in long-term follow up following stereotactic radiotherapy

R Locke, G Kontorinis, J Crowther

From the Southern General Hospital, Glasgow

\section{Background and objective}

Stereotactic radiosurgery is a well-recognised treatment for growing or medium-sized vestibular schwannomas. Although most vestibular schwannomas will respond positively to stereotactic radiosurgery, the expected change in size is unknown. This study aimed to quantify the growth patterns after stereotactic radiosurgery.

\section{Methods}

A retrospective analysis of patients managed in a West of Scotland tertiary referral centre was performed. The tumour of each patient was measured on axial, contrast-enhanced, T1-weighted magnetic resonance imaging conducted before treatment and one year after stereotactic radiosurgery and on the most recent scan. A significant change in size was defined as greater than $1 \mathrm{~mm}$.

Results

Over a one-year period, 30 per cent of patients underwent stereotactic radiosurgery. Thirty-nine per cent had no 
significant change in tumour size, 48 per cent showed a decrease in size and 13 per cent showed an increase in size. Overall, there was a mean reduction of $0.2 \mathrm{~mm}$ per year following stereotactic radiosurgery. This increased to $1 \mathrm{~mm}$ per year when we measured only the tumours that responded positively to stereotactic radiosurgery.

\section{Conclusion}

Our results show a trend for a decrease in tumour size in long-term follow up for most patients receiving stereotactic radiosurgery. For those vestibular schwannomas that respond positively to stereotactic radiosurgery, this decrease rate is significantly higher. 